\title{
Endoparasitos em veados-catingueiros (Mazama gouazoubira) mantidos em cativeiro no Semiárido Paraibano*
}

\author{
Endoparasites in brown brocket deer (Mazama gouazoubira) \\ kept in captivity in the Semiarid of Paraíba
}

\author{
Claudia Soares Dantas, ${ }^{* *}$ Jossiara Abrante Rodrigues, ${ }^{* * *}$ Vinícius Longo Ribeiro Vilela**,***
}

\begin{abstract}
Resumo
Objetivou-se investigar a ocorrência de endoparasitos em veados-catingueiros (Mazama gouazoubira) pertencentes a uma propriedade conservacionista no município de Sousa, Paraíba. Foram realizadas coletas de fezes em sete animais que foram submetidas às técnicas de sedimentação espontânea, centrífugo-flutuação em solução de sacarose, contagem de ovos por grama de fezes (OPG), contagem de oocistos por grama de fezes (OoPG) e coproculturas. Foram avaliados os locais de criação dos animais, para obtenção das informações acerca do manejo sanitário e alimentar. Em todas as amostras observaram-se ovos da Superfamília Trichostrongyloidea (OPG $200 \pm 100$ ) e coccídios (OoPG $1500 \pm 300$ ). Nas coproculturas, 100\% das larvas infectantes pertenciam ao gênero Trichostrongylus. Foram constatados oocistos do gênero Cystoisospora em todas as amostras. Em uma fêmea, que apresentou OoPG 1800, observaram-se sinais clínicos como apatia, anorexia, desidratação e diarreia intermitente, condizentes com Coccidiose. Este animal recebeu tratamento com a associação de sulfametoxazol e trimetropim, por sete dias, havendo melhora clínica. Erros de manejo foram observados na propriedade, como o convívio de M. gouazoubira com bovinos, ovinos e caprinos, alimentação inadequada à base de ração industrializada para equinos, bebedouros improvisados e não higienizados, e acúmulo de sujeira dos ambientes frequentados pelos animais. Concluiu-se que os veados-catingueiros estavam parasitados por helmintos (Trichostrongylus sp.) e protozoários (Cystoisospora spp.), podendo estas infecções estarem associadas ao convívio destes animais com ruminantes domésticos. Este estudo apresenta o primeiro relato da infecção por Cystoisospora spp. em M. gouazoubira.
\end{abstract}

Palavras-Chave: Cervídeos, Cistoisosporose, Coccidiose

\begin{abstract}
The objective of this study was to investigate the occurrence of endoparasites in brown brocket deer (Mazama gouazoubira) belonging to a conservationist property in the municipality of Sousa, Paraíba. Fecal samples were collected in seven animals that were submitted to the techniques of spontaneous sedimentation, centrifugation-flotation in sucrose solution, egg count per gram of feces (EPG), oocyst count per gram of feces (OoPG) and coproculture. The breeding sites were evaluated to obtain information on Sanitary and food management. In all samples, eggs of the Trichostrongyloidea Superfamily (EPG 200 \pm 100 ) and coccidia (OoPG $1500 \pm 300$ ) were observed. In the coprocultures, $100 \%$ of the infecting larvae belonged to the genus Trichostrongylus. Oocysts of the genus Cystoisospora spp. were found in all samples. In one female, who presented OoPG 1800, clinical signs such as apathy, anorexia, dehydration and intermittent diarrhea, consistent with Coccidiosis. This animal received treatment with the combination of sulfamethoxazole and trimetropim for seven days, with clinical improvement of the animal. Management errors were observed in the property, such as M. gouazoubira's conviviality with as cattle, sheep and goats, inadequate feed based on industrialized ration for horses, improvised and unhygienic drinking fountains, accumulation of dirt in the environments frequented by animals. They concluded that veados-catingueiros were parasitized by helminths (Trichostrongylus sp.) and protozoa (Cystoisospora sp.) infections may be associated with the coexistence of animals with domestic ruminants. This study presents the first report of Cystoisospora spp. in M. gouazoubira.
\end{abstract}

Keywords: Cervidae, Cystoisosporosis, Coccidiosis

${ }^{*}$ Recebido em 26 de maio de 2019 e aceito em 6 de novembro de 2019.

**Departamento de Medicina Veterinária, Instituto Federal da Paraíba, IFPB, campus Sousa, Paraíba, Brasil;

***Programa de Pós-Graduação em Ciência Animal, Universidade Federal de Campina Grande, UFCG, campus Patos, Paraíba, Brasil;

Autor para correspondência. Av. Pres. Tancredo Neves, s/n, Jardim Sorrilândia, Sousa-PB. CEP: 58800-970. E-mail: vinicius.vilela@ifpb.edu.br 


\section{Introdução}

O veado-catingueiro (Mazama gouazoubira) é uma pequena espécie de cervídeo, pertencente à ordem Artiodactyla, pesando em torno de $20 \mathrm{~kg}$, com altura média na cernelha de $50 \mathrm{~cm}$. A coloração geral dos indivíduos é extremamente variável, podendo ir do cinza escuro até o marrom avermelhado (DUARTE et al., 2012). Os machos possuem chifres pequenos e simples, com cerca de sete centímetros de altura (MARQUES et al., 2007). É o cervídeo mais abundante na América do Sul, presente em uma vasta diversidade de habitats. No Brasil, pode ser encontrado desde áreas antropizadas, até cerrado fechado, ou áreas ocupadas pela agricultura, sendo uma espécie que se adapta a ambientes modificados (VOGLIOTTI, 2003; DUARTE et al., 2006).

Uma consequência da aproximação entre ruminantes silvestres, principalmente $M$. gouazoubira e ruminantes domésticos, é o intercâmbio de patógenos entre suas populações. Com isso, o conhecimento relacionado aos microrganismos infecciosos é importante para que cervídeos mantidos em cativeiro não sejam portadores, nem carreadores de patógenos, no que diz respeito ao trânsito de animais entre zoológicos e estações de reprodução (SILVEIRA et al., 2011).

Os estudos sanitários com cervídeos silvestres restringem-se a observações em zoológicos, parques conservacionistas ou parques criatórios (BARRY et al., 2002), no entanto, estudos sobre endoparasitas nestas espécies de animais são escassos. Segundo Marques et al. (2007), as doenças parasitárias são consideradas as principais causas de doença e mortalidade, possivelmente, provindas do contato com animais domésticos e a falta de higienização dos recintos onde estão alojados.

Em estudo realizado por Nascimento et al. (2000), na região do Pantanal, ao analisarem material de M. gouazoubira, constataram as espécies Trichostrongylus axei, T. colubriformis, Cooperia pectinata, C. punctata, Monodontus sp., Haemonchus contortus, $\mathrm{H}$. similis. No trabalho realizado por Marques et al. (2007), foram coletadas fezes de M. gouazoubira em uma reserva no estado de Santa Catarina. Nessas amostras, oocistos de protozoários do gênero Eimeria spp. e ovos de trematódeos do gênero Paramphistomum foram identificados. Embora apresentando oocistos e ovos dos parasitos, os animais não apresentavam sinais clínicos que caracterizassem uma doença parasitária.

A escassez de informações sobre os principais parasitos desses animais dificulta o estabelecimento de medidas de controle e profilaxia para as enfermidades parasitárias. Portanto, o objetivo deste estudo foi identificar endoparasitos gastrintestinais de veados-catingueiros ( $M$. gouazoubira) mantidos em reserva conservacionista no município de Sousa, Paraíba.

\section{Material e métodos}

Esta pesquisa recebeu autorização de execução pelo Sistema de Autorização e Informação em Biodiversidade - SISBIO (Registro $n^{\circ} 68549-1$ ) para a coleta de material biológico e para a realização de pesquisa em unidade fora de conservação federal.

Foram realizadas visitas em uma propriedade conservacionista localizada no Município de Sousa, Paraíba. O interior do Nordeste brasileiro pertence ao bioma Caatinga, que compreende $12 \%$ do território do Brasil. O clima é caracterizado como quente e semiárido, com temperaturas médias de $27^{\circ} \mathrm{C}$ ao longo do ano, e pluviosidade média anual de aproximadamente $500 \mathrm{~mm}^{3}$. Apresenta tipicamente duas estações: chuvosa, de janeiro a maio, e seca, de junho a dezembro (BATISTA et al., 2007).

A propriedade apresentava sete veados-catingueiros $(M$. gouazoubira), sendo um macho adulto, três fêmeas adultas e três filhotes. Os animais estavam em um espaço de 0,2 ha, onde algumas áreas estavam com muita umidade. Não era realizada a limpeza diária, apresentando acúmulo de fezes e de restos de alimentos. Ainda, o recinto era delimitado por cerca de arame, sendo circunvizinho a outros recintos onde habitavam animais de espécies tanto silvestres, como porcos do mato (Tayassu tajacu), emas (Rhea americana), cutias (Dasyprocta spp.), além de várias espécies de pássaros migratórios e nativos da região, como também animais domésticos, a exemplo de ovinos, caprinos e bovinos, não havendo corredor de isolamento entre eles.

Foram coletadas amostras fecais dos M. gouazoubira diretamente da ampola retal e armazenadas em sacos coletores, acondicionadas em caixa térmica com gelo e submetidas para análises no Laboratório de Parasitologia Veterinária (LPV) do Instituto Federal da Paraíba, campus Sousa.

Foram realizados os métodos de sedimentação espontânea, de acordo com Hoffmann, Pons e Janer (1934), contagem de ovos por grama de fezes (OPG) e contagem de oocistos por grama de fezes (OoPG), de acordo com Gordon e Whitlock (1939), e Coproculturas, conforme Roberts e O'Sullivan (1950).

Para a esporulação de coccídios, adicionou-se dicromato de potássio $2,5 \%$, sendo as amostras acondicionadas em estufa B.O.D. a $28^{\circ} \mathrm{C}$, por 72 horas. Após esse período, foram realizadas novas análises fecais para a busca de protozoários entéricos, procedendo-se a técnica de centrífugo-flutuação em solução de sacarose (BOWMAN et al., 2010). A análise morfométrica dos coccídios foi realizada após a esporulação, utilizando microscópio MAX-300 ${ }^{\circledR}$ com objetivas de 10X e 40X, associado a um computador instalado com o programa $\mathrm{Mv}$ Image $^{\circledR}$.

Foram aferidos o diâmetro polar (máximo, mínimo e médio), diâmetro equatorial (máximo, mínimo e médio), índice morfométrico e a espessura da parede do oocisto, de acordo com as características morfológicas dos oocistos esporulados descritas por Daugschies et al. (2004).

Foi preenchido um formulário epidemiológico para a coleta de informações referentes ao manejo sanitário e alimentar dos animais, a fim de traçar estratégias de controle e profilaxia para a correção dos casos de diarreia.

\section{Resultados e discussão}

$\mathrm{Na}$ propriedade de estudo os veados-catingueiros tinham acesso a outros animais de espécies tanto silvestres, como também animais domésticos, a exemplo de ovinos, caprinos e bovinos, não havendo corredor de isolamento entre eles. Segundo Beltrán-Saavedra et al. (2009), o convívio de animais domésticos e silvestres facilita a introdução e propagação de doenças entre essas espécies, podendo comprometer a sanidade dos mesmos. 
As amostras fecais de todos os animais foram positivas para a superfamília Trichostrongyloidea, com média de OPG de $200 \pm 100$. De acordo com Duarte et al. (2006), as infecções helmínticas mais importantes localizam-se no trato gastrintestinal, sendo constituídas, na sua maioria, por tricostrongiloidose e estrongiloidose.

Nas coproculturas, as larvas foram identificadas morfologicamente de acordo com as características descritas por Keith (1953), onde $100 \%$ das larvas infectantes pertenciam ao gênero Trichostrongylus. Entre os cervos, bem como nos ruminantes domésticos, a superfamília Trichostrongyloidea, é a mais amplamente difundida, podendo causar sinais clínicos severos ou até mesmo nenhum sinal (OROZCO et al., 2013). Apesar de o gênero predominante ter sido Trichostrongylus, o nível de infecção dos animais estava baixo. Quando se aplica o conhecimento para caprinos e ovinos, de acordo com Molento et al. (2009), infecções helmínticas inferiores a 500 OPG são consideradas leves. Um fator que pode ter favorecido a esse baixo nível de helmintos gastrintestinais foi o manejo anti-helmíntico realizado na propriedade, com administrações periódicas de cloridrato de levamisol $5 \%(5 \mathrm{mg} / \mathrm{kg})$, duas aplicações via oral com intervalos de 21 dias.

Pelo OoPG, foi observada a presença de oocistos de coccídios em todas as amostras, com infecção média de $1500 \pm 300$. Após a esporulação dos oocistos, foi constatada microscopicamente a presença do gênero Cystoisospora (Figura 1), observaram-se coccídios cuja morfologia apresentava dois esporocistos, cada qual com quatro esporozoítos em seu interior. Vinte coccídios tiveram suas dimensões mensuradas, revelando valores médios (em $\mu \mathrm{m})$ de diâmetro polar máximo $(21,314)$, mínimo $(16,715)$ e médio (18士); diâmetro equatorial máximo (14,430), mínimo $(11,203)$ e médio $(13 \pm)$; índice morfométrico $(1,47)$, grânulo polar ausente e parede lisa, o que diferiu morfologicamente Cystoisospora dos demais gêneros de coccídios.

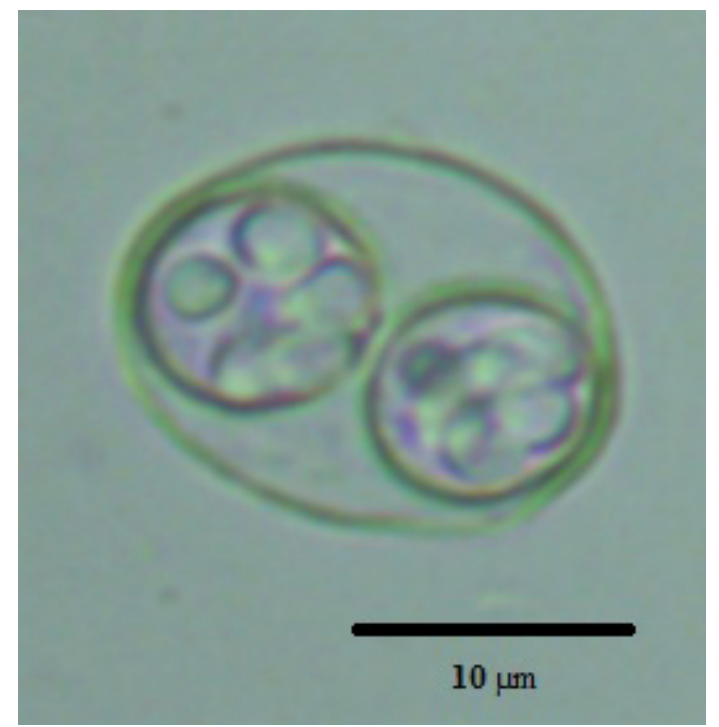

Figura 1: Oocisto esporulado de Cystoisospora spp. diagnosticado em fezes de veados-catingueiros (M. gouazoubira) no Semiárido brasileiro.
Em um animal, fêmea, adulta, aproximadamente dois anos de idade, foi observada magreza, apatia, inapetência, desidratação e histórico de diarreia intermitente há dez dias, sinais clínicos condizentes com coccidioses (Figura 2). O animal vinha sendo medicado com cloridrato de levamisol $5 \%$ (5mg/ kg, duas doses), sem melhora do seu quadro clínico. Foi separada dos demais para realização da avaliação clínica específica, onde se verificou que não havia sinais de ectoparasitos e mucosas pouco coradas.

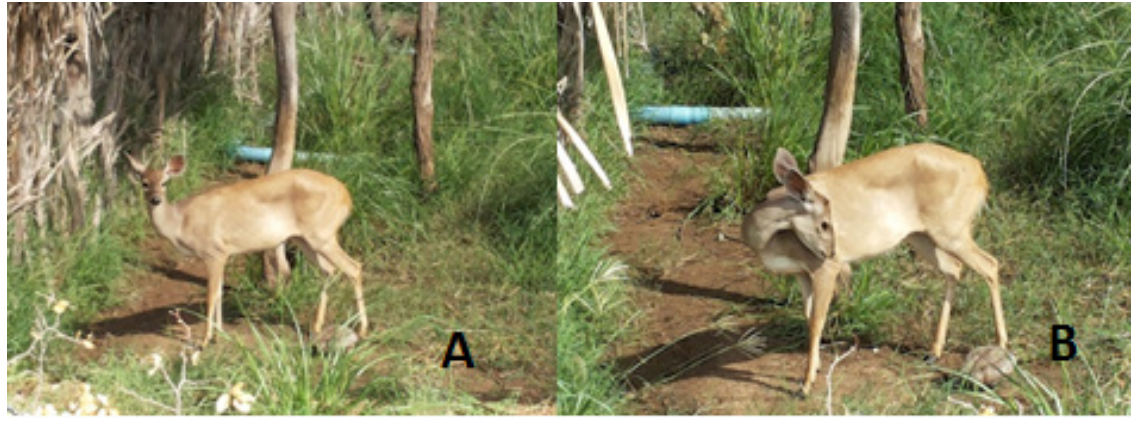

Figura 2. Fêmea de M. gouazoubira apresentando sinais clínicos de magreza (A) e apatia (B), em propriedade conservacionista no Semiárido do Brasil

De acordo com Endo et al. (2014), as enfermidades parasitárias podem ser diagnosticadas por meio dos sinais clínicos, associados aos exames laboratoriais, dados epidemiológicos e achados de necropsia. Desta forma, por apresentar alta infecção por este parasito (OoPG 1800 ) e sintomatologia condizente, este animal foi diagnosticado com Cistoisosporose.

Vilela et al. (2017) relataram pela primeira vez o parasitismo por Cystoisospora spp. em búfalos, no Brasil. Entretanto, não há relatos em pequenos ruminantes, tão pouco em cervídeos silvestres, sendo este o primeiro relato da infecção por Cystoisospora spp. em M. gouazoubira.

Com relação ao manejo dos animais, foram observados vários erros na propriedade, como o fornecimento de ração industrializada para equinos em cochos improvisados, frutas em decomposição ofertadas no chão, bebedouro inapropriado e sem higienização, e o espaço onde viviam era insuficiente para comportar os animais (Figura 3).

Algumas áreas onde os animais frequentavam estavam com muita umidade, sem nenhuma cama ou proteção. $A$ sobrevivência dos coccídios no ambiente aumenta quando encontra condições favoráveis de temperatura, umidade e oxigenação. Criações com manejo inadequado, ausência de higiene e animais aglomerados facilitam a disseminação das espécies de Cystoisospora (FILHO et al., 2010).

Com o resultado dos exames, nova visita foi efetuada na propriedade para sugestão das correções dos erros no manejo sanitário e alimentar, de acordo com as orientações de Duarte et al. (2006). Foi instituído o tratamento para coccidiose, utilizando sulfametoxazol com trimetoprim $(50 \mathrm{~g} / 50 \mathrm{~L})$, diluído em água, durante sete dias. Após dez dias da instituição do tratamento outra visita foi feita e o animal apresentava-se recuperado de sua sintomatologia clínica, amostras foram coletadas de todos os animais, para realização da OoPG e todos apresentavam-se negativos para coccídios, resultando em um tratamento efetivo. 


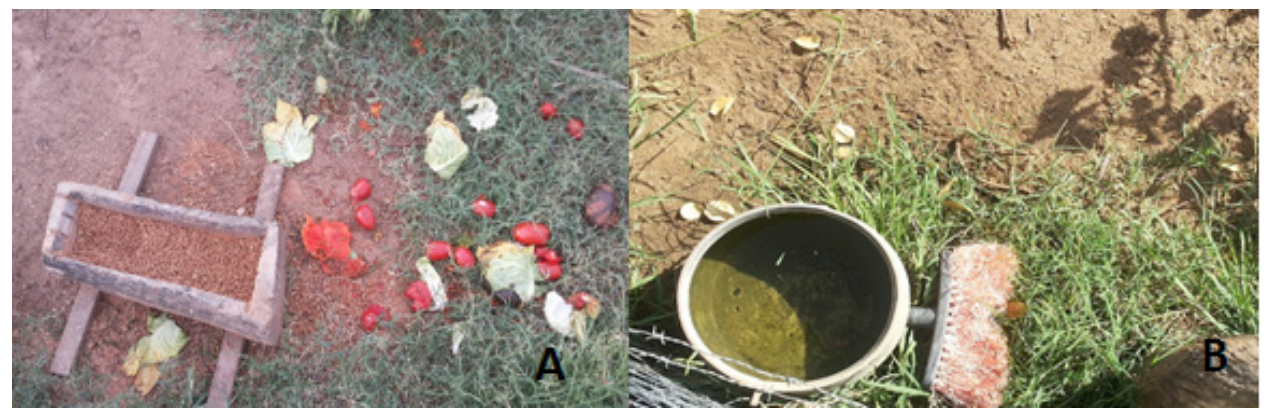

Figura 3. Alimentação (A) e oferta de água (B) inadequada oferecida aos veados-catingueiros (M. gouazoubira) em propriedade conservacionista no Semiárido brasileiro

\section{Conclusão}

Os veados-catingueiros esta-vam parasitados por helmintos (Trichostrongylus sp.) e proto-zoários (Cystoisospora spp.),

\section{Referências}

ANTUNES, V.S. Área de uso e seleção de habitats por veadocatingueiro (Mazama gouazoubira; Fisher, 1814) no Pantanal da Nhecolândia-MS. Dissertação de Mestrado. Escola Superior de Agricultura, Universidade de São Paulo, Piracicaba, 74p, 2012.

BARRY, T.N.; HOSKIN, S.O.; WILSON, P.R. Novel forages for growth and health in farmed deer. New Zeland Veterinary Journal, v.50, n.6, p.244-251, 2002.

BATISTA, J.S.; RIET-CORREA, F.; TEIXEIRA, M.M.G.; MADRUGA, C.R.; SIMÕES, S.D.V.; MAIA, T.F. Trypanosomiasis byTrypanosoma vivax in cattle in the Brazilian semiarid:

Description of an outbreak and lesions in the nervous system. Vet. Parasitol. 143:174-181, 2007.

BELTRÁN-SAAVEDRA, L.F.; ANGULO, S.; GONZALES, J.L. Uso de metodologías de censos muestra les indirectos de fecas para evaluar endoparasitos em mamíferos silvestres: Um ensayo en la Reserva Privada de San Miguelito, Santa Cruz, Bolivia. Ecologia em Bolivia, v. 44, n. 1, p. 56-61, 2009.

BOWMAN, D.D.; LYNN, R.C.; EBERHARD, M.L. Parasitologia Veterinária de Georgis. 9 ed. São Paulo: Manole, 2010, p. 115-201.

DAUGSCHIES, A.S; IMARON, M.; GANTER, M.; BOLLWAHN, W. Prevalence of Eimeria spp. in sows at piglet-producting farms in Germany, J Vet Medicine, v. 51, p. 135-139, 2004.

DUARTE, J.M.B. Artiodactyla - Cervidae (Veados e Cervos). In: CUBAS, Z.S.; SILVA, J.C.R.; CATÃO-DIAS, J.L. Tratado de animais selvagens. São Paulo: Roca; 2006. p. 1354.

DUARTE, J.M.B.; VOGLIOTTI, A.; ZANETTI, E.S.; OLIVEIRA, M.L.; TIEPOLO, L.M.; RODRIGUES, L. F.; ALMEIDA, L.B. Avaliação de Extinção do Veado Catingueiro Mazama gouazoubira G. Fischer [von Waldheim], 1814, no Brasil. Biodiversidade Brasileira. Ano II, nº 3, p. 50-58, 2012.

ENDO, V.T.; OLIVEIRA, T.C.; CABRAL, A.P.M.; SAKAMOTO, K.A.M.; FERRARO, C.G.; PEREIRA, V.; LOPES, W.D.Z.; MAZZUCATTO, B.C. Prevalência dos helmintos Haemonchus contortus e Oesophagostomum columbianum em pequenos ruminantes atendidos no setor de Anatomia Patológica - UEM. Revista de Ciência Veterinária e Saúde Pública, v. 1, n. 2, p. 112114, 2014. podendo estas infecções estarem associadas ao convívio destes animais com ruminantes domésticos. Este estudo representa o primeiro relato da infecção por Cystoisospora spp. em M. gouazoubira.

FILHO, W.L.T; CARDOZO, S.V.; LOPES, C.W.G. Viabilidade e morfobiologia dos oocistos de Cystoisospora ohioensis (DUBEY, 1975) Frenkel, 1977 (Apicomplexa: Cystoisosporinae) Eliminados por cães infectados experimentalmente. Rev. Bras. Med. Vet., 32(3):161-165, jul/set 2010.

GORDON, H.M.; WHITLOCK, H.V. A new technique for counting nematode eggs in sheep faeces. J. Coun. Sci. Ind. Res. 12, 50-52, 1939.

HOFFMANN, W.A.; PONS, J.A.; JANER, J.L. Sedimentation concentration method in schistosome. Puerto Rico Journal of Public Health and Tropical Medicine, 1934.

MARQUES, S.M.T.; QUADROS, R.M.; MAZZOLLI, M.; JESUS, J.R. Parasitos gastrintestinais em veados (Mazama gouazoubira) de áreas nativas no Planalto de Santa Catarina, Brasil. Veterinária em Foco, v. 5, p. 3-9, 2007.

KEITH, R.K. The differentiation of infective larvae of some common nematode parasites of cattle. Australian Journal of Zoology, v. 1, n. 2, p. 223-235, 1953.

MOLENTO, M.B.; GAVIÃO, A.A.; DEPNER, R.A.; PIRES, C.C. Frequency of treatment and production performance using the FAMACHA method compared with preventive control in ewes. Veterinary Parasitology, v.162, n.3/4, p.314-319, 2009.

NASCIMENTO, A.A.; BONUTI, M.R.; MAPELI, E.B.; TEBALDI, J.H.; ARANTES, I.G.; ZETTERMANN, C.D. Infecções naturais em cervídeos (Mammalia: Cervidae) procedentes dos Estados do Mato Grosso do Sul e São Paulo, por nematódeos Trichostrongyloidea Cram, 1927. Braz. J. Vet. Res. Anim. Sci., São Paulo, v. 37, n. 2, 2000.

OROZCO, M.M.; MARULL, C.; JIMÉNEZ, I.; GURTLER, R.E. Mortalidad invernal

de ciervo de los pantanos (Blastocerus dichotomus) em humeda les del noreste de Argentina. Mastozoologia Neotropical, v. 20, n. 1, p.163-170, 2013.

ROBERTS, F.H.S.; O'SULLIVAN, J.P. Methods for egg counts and larval cultures for strongyles infesting the gastrointestinal tract of cattle. Aust. Agric. Rec. p. 199-102, 1950. 
SILVEIRA, J.A.G.; RABELO, E.M.L.; RIBEIRO, M.F.B. Detection of Theileria and Babesia in brown brocket deer (Mazama gouazoubira) and marsh deer (Blastocerus dichotomus) in the State of Minas Gerais, Brazil. Veterinary Parasitology, v. 177, p. 61-66, 2011.

URQUHART, G.M.; ARMOUR, J.; DUNCAN, J.L.; DUNN, A.M.; JENNINGS, F. W. Parasitologia Veterinária. 2 ed. Rio de Janeiro: Koogan, 1998, 273p.
VILELA, V.L.R.; FEITOSA, T.F.; BRASIL, A.W.L.; PARENTONI, R.N.; R. A. BEZERRA, R.A.; AZEVÊDO, S.S.. Prevalence of gastrointestinal parasites in buffaloes in the state of Paraiba and the first report of Cystoisospora spp. in buffaloes in Brazil. ARS VETERINARIA, Jaboticabal, SP, v.33, n.1, 026-030, 2017.

VOGLIOTT, A. História natural de Mazama bororo (Artiodactyla; Cervidae) através de etnozoologia, monitoramento fotográfico e rádio-telemetria. Dissertação de mestrado, Escola Superior de Agricultura Luiz de Queiroz USP, Piracicaba, 113 p, 2003. 